\title{
Harmonics-Based Bio-Implantable Telemetry System
}

\author{
Anthony N. Laskovski, Student Member, IEEE and Mehmet R. Yuce, Member, IEEE
}

\begin{abstract}
Miniaturization is a key focus for medically implantable electronics such as Cochlear and Retinal prosthesis, and medical monitoring and recording applications. The need for low power dissipation is equally important, and powerhungry crystals and oscillators are commonly used to produce and control the implant's carrier transmission frequency. This paper presents a new harmonics-based method that allows this transmission frequency to be varied and controlled externally, while also minimizing the size and power requirements of certain implanted devices.
\end{abstract}

\section{INTRODUCTION}

The use of implantable wireless electronics for medical monitoring, treatment and diagnosis is indeed exciting, especially considering the rapid rate at which electronics technology advances. Developments in this field enable patients with freer movement, and equip medical staff with more sophisticated processing and communication of medical information.

Electronic design generally involves many constraints, which require devices to achieve a certain level of complexity and use a certain amount of power while fitting within a certain space. When one considers the application of electronics to the medically implantable level, these design conditions are further tightened, in addition to a number of medical safety constraints [1]. The need to achieve higher efficiency, smaller size and higher data rates for more complex devices is evident.

Considerable progress has been made in this area with examples such as the application of the Class-E amplifier for power transmission [2], the development of implanted battery charging [3], [4], the optimization of coil geometry and transmission frequency and the use of dual band wireless power and data [5], [6]. If power and data are sent at lower and higher frequencies respectively, power is transferred more efficiently, and data may be sent at a higher rate. One point which is common throughout most of these technologies, is that the implanted devices generally consist of a power receiving coil, a DC power supply, a self-oscillating unit, data/telemetry and transmitter/modulator unit.

Harmonic components are often avoided in electronics design, generally treated as unwanted noise and minimized in many situations so as to avoid disrupting the intended function of the circuit. Most analyses related to harmonics are derived to account for possible errors in a circuit's performance. However, as this paper will elaborate, harmonics

This work was supported in part by the Australian Research Council (ARC) under the Discovery Projects Grant.

A. N. Laskovski and M. R. Yuce are with the School of Electrical Engineering and Computer Science, University of Newcastle, Callaghan, NSW 2308, Australia anthony.laskovski@newcastle.edu.au, mehmet.yuce@newcastle.edu.au
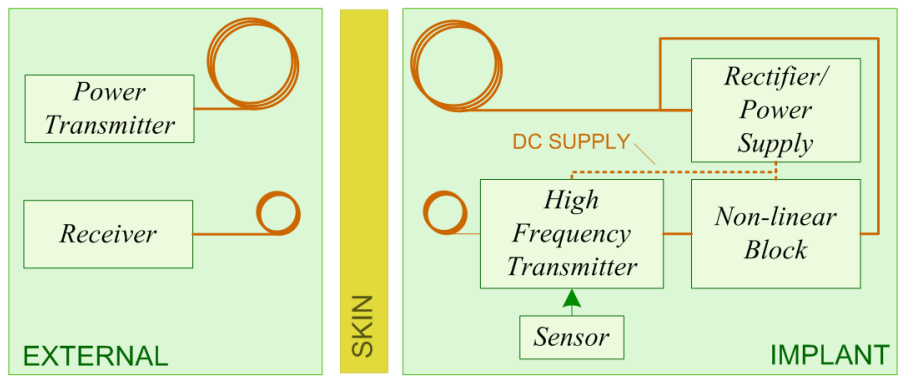

Fig. 1. A Block diagram to represent the internal and external device architecture.

may also be considered useful, adding a new elegance to implantable electronics design.

\section{Proposed Architecture}

The architecture proposed in this paper begins with the idea that power and data signals will be sent at lower and higher frequencies respectively. This means that while power is wirelessly sent to the implant as shown in Fig. 1, a high frequency carrier signal must be generated within the implant to send the data back to an external receiver, which is optimized at the higher frequency.

The system in Fig. 1 does not consist of a power-hungry crystal nor an oscillator to generate a data carrier within the implant. In the proposed system, the data carrier-frequency is prodyced by generating harmonics from the received power signal itself. This means that while the lower-frequency power signal is used to generate DC power for the implant, it is also used to generate higher frequency harmonics by using its oscillatory nature. This is why the incoming signal from the power coil is shown to branch off to two units in the implanted device: One to the Radio Frequency (RF) AC to DC rectifier, and one to the Non-Linear block.

\section{A. Non-Linear Block}

The Non-Linear block in Fig. 1 aims to produce harmonic frequencies by using both the received power signal and a DC power supply which was also derived from the same signal. A representation using the Fourier transform will illustrate the concept.

Essentially, any signal may be mathematically represented as an infinite sum of pure sine waves at different frequencies. If a periodic saw-tooth signal is considered as an example, the signal may be visualized as a combination of many sine waves, each at different frequencies and power levels. They sum together to create what is seen as a saw-tooth wave. 


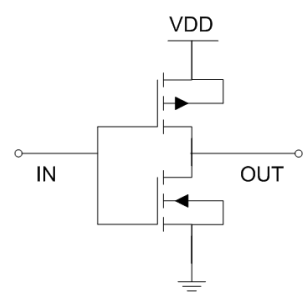

Fig. 2. Inverter: A non-linear block

If one of the constituent frequencies is removed, the sawtooth wave will no longer hold its saw-tooth shape. It means that any non-sinusoidal wave may have many sinusoidal harmonic frequencies which can be potentially useful in RF circuits.

It is useful to further consider the harmonic characteristics of a commonly known wave shape in electronics, the square wave or periodic pulse signal. This signal has two voltage levels, low and high. It changes levels periodically, and the duty cycle of the signal represents the proportion of time that the signal is high compared to low within the signal period. A mathematical representation of the Fourier series for the periodic square pulse signal was expressed in [7] as

$$
x(t)=\frac{A \tau}{T}+\frac{2 A \tau}{T} \sum_{k=1}^{\infty} \frac{\sin \left(\pi k \frac{\tau}{T}\right)}{\pi k \frac{\tau}{T}} \cos \left(2 \pi k \frac{t}{T}\right)
$$

where $\tau$ is the pulse width in seconds, $T$ is the period of the signal, $A$ is the amplitude, $k$ is the integer harmonic level and $t$ is time. From this equation, it is evident that by manipulating the duty cycle $(\tau / T)$ the distribution of harmonics can be changed. Generally speaking, as the duty cycle decreases and the pulses become sharper, more sinusoidal harmonics exist at higher frequencies.

Consider the incoming power signal from the power coil. It is a purely sinusoidal signal, frequency $\omega_{p}$. If this signal is converted to a $0.5 \%$ square-wave, the resultant signal will no longer be a simple sine wave but a combination of many different frequencies $\omega_{p}, 3 \omega_{p}, 5 \omega_{p} \ldots$ as shown in (2). This means that extracting one of the harmonics for data communications is as simple as filtering the output of the non-linear block of the system and using the result as the data carrier. It should also be noted that the non-linear block may be implemented by a range of circuits such as inverters, comparators, diodes etc. A sample non-linear block is presented in Fig. 2.

$x(t)=\frac{A}{2}+A\left(\frac{2}{\pi}\left(\cos (\omega t)-\frac{2}{3 \pi} \cos (3 \omega t)+\frac{2}{5 \pi} \cos (5 \omega t) \ldots\right)\right.$

This approach is elegant for a number of reasons. It uses the incoming power signal to generate a higher frequency data carrier in addition to producing DC power. It does so by non-linearizing a sinusoidal signal, and it eliminates the need for self oscillating devices such as crystals or voltage controlled oscillators within the implant, which require large inductors and capacitors. It decreases the size of the circuitry in the implant, and it only requires the sinusoidal input power

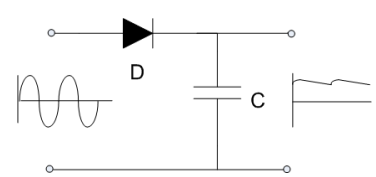

Fig. 3. A diode and capacitor rectifier

signal and a connection to the DC rectifier's power, which is also produced from the same signal.

\section{B. Rectifier/Power Supply}

The power supply unit in Fig. 1 serves the purpose of converting the RF input signal from $\mathrm{AC}$ to $\mathrm{DC}$ and possibly storing the energy in a rechargeable battery. There are many ways in which this can be achieved, however it is important to consider that DC rectification becomes less conventional when the frequency increases to $\mathrm{MHz}$ range and transmission voltage decreases such that something as simple as a diode voltage drop may determine the difference between successfully producing DC power or not.

The rectifier which was used in this specific implementation was chosen for its minimal number of components, comprising a diode and capacitor as shown in Fig. 3. The diode provides half-wave rectification, while the capacitor stores charge and releases the energy during the diode's offphase.

\section{Internal Transmitter}

The transmitter involves using the high frequency carrier produced by the Non-Linear block to transmit data to an external receiving device, and it may employ various topologies depending on particular system restrictions. The recently established Medical Implant Communication Service (MICS) standard specifies a frequency range between $402 \mathrm{MHz}$ and $405 \mathrm{MHz}$, with a maximum bandwidth of $300 \mathrm{kHz}$ at any time and a maximum transmission power of $25 \mu \mathrm{W}$ or $-16 \mathrm{dBm}$ [1]

Given that the power requirements of this standard are so low, the transmitter implemented in this paper simply involved a Band Pass Filter as shown in Fig. 4, which serves two purposes. It reduces or filters the unused harmonics, allowing the $403 \mathrm{MHz}$ MICS frequency through. Secondly, if $v_{\text {out }}$ is considered as the voltage across the inductor of the circuit, it can also represent the coil antenna from which the high frequency data signal is transmitted. Modulated data can also be transmitted using this topology depending on its modulation scheme. A simple On Off Keying (OOK) scheme can be achieved by placing a transistor switch in the series RLC circuit, which is controlled by the digital data signal, supplied from a sensor. Other modulation schemes may be implemented, depending on the filter/transmission network.

The transfer function of the bandpass filter is given by (3). Given the fact that it is a second order system, it can be designed in mind of damping and $\mathrm{Q}$ factors.

$$
H(s)=\frac{v_{\text {out }}}{v_{\text {in }}}=\frac{s^{2}}{s^{2}+s \frac{R}{L}+\frac{1}{L C}}
$$




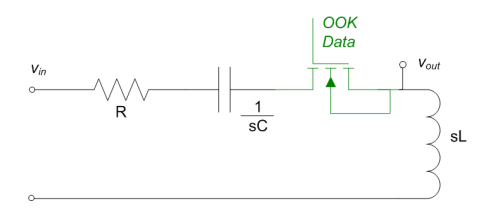

Fig. 4. Bandpass filter transmitter

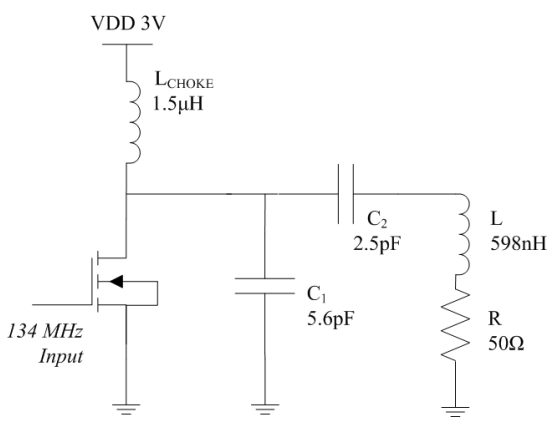

Fig. 5. The Class-E power transmitter at $134 \mathrm{MHz}$

\section{HARDWARE MEASUREMENTS}

Ideas are often theoretically dreamt up and simulated, however until a working example has been tested the interesting concepts can remain only theoretical. This is especially true in RF electronics, and for this reason, an example of the proposed architecture was successfully implemented in hardware.

The MICS standard was used as the basis of the data transmission, which means that the data signal's frequency was restricted from $402-405 \mathrm{MHz}$ with a transmission power less than $-16 \mathrm{dBm}$. Working backwards from this frequency by using the discussion in Section II-A, one may tune the frequency of the power transmission externally. Since third harmonics possess the highest power (2) it was decided that for this hardware test, the fundamental power signal would be selected such that $403 \mathrm{MHz}$ would represent the third harmonic of the power signal. This made the desired power transmission frequency $134-135 \mathrm{MHz}$. It is important to note this technique allows the frequency of data transmission to be tuned, varied and controlled externally, meaning that once an implant has been implanted, its transmission frequency can be altered according to the environment or transmission standard.

\section{A. External Power Transmitter}

The power transmitter was designed as a Class-E amplifier optimized at around $134 \mathrm{MHz}$. The circuit components were selected using equations from [8] and the circuit was constructed as shown in Fig. 5, where $L$ represents the inductive power link. The measured output waveform of this transmitter is presented in Fig. 6 with a $V_{\text {peak }}$ close to $1.5 \mathrm{~V}$.

\section{B. Internal DC Supply}

The recctification circuit of Fig. 3 was constructed to serve as the implant's DC power supply. The energy was not rectified to charge a battery in this instance, but to be

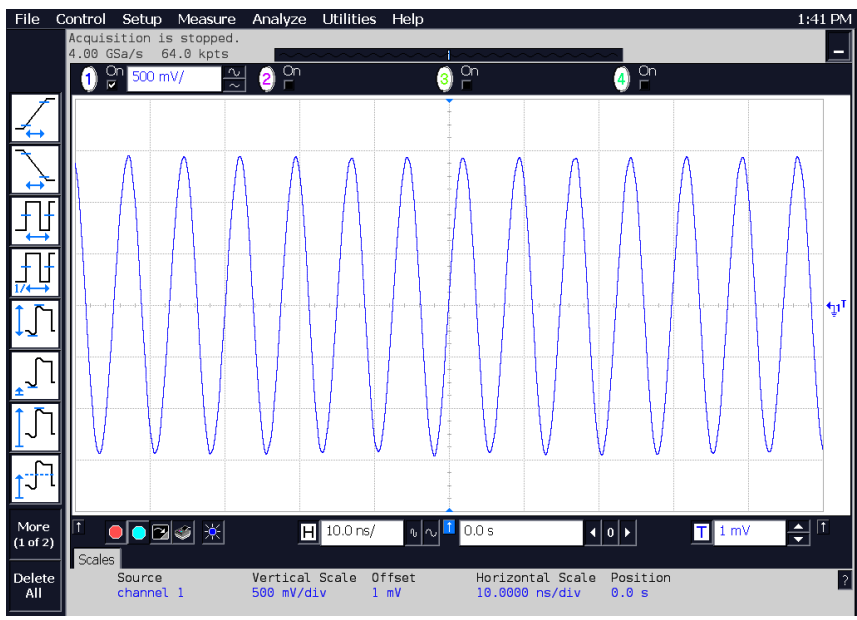

Fig. 6. Output of the $134-5 \mathrm{MHz}$ Class-E power transmitter

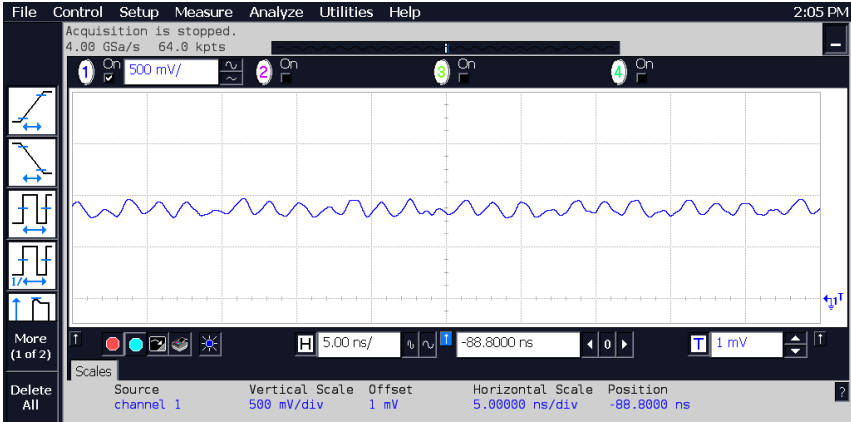

Fig. 7. Measured Output signal of the DC power supply

instantaneously used. The reason why a half wave rectifier was used in this specific implementation is because it minimized the diode bias voltage drop and the number of circuit elements. The capacitor $C$ had a value of $6.6 \mu \mathrm{F}$, and the output waveform is displayed in Fig. 7 as around 1V.

\section{Non-Linear Block}

The implementation of this unit involved using an inverter MOSFET chip, which was powered by the DC power obtained from the internal power supply. The power receiver coil was connected directly to the inverter chip and the output of the inverter was represented on a Spectrum Analyser and presented in Fig. 8.

\section{Transmitter}

The transmitter was implemented as the band-pass filter of Fig. 4, as the signal level coming out of the inverter was high enough to transmit within the MICS standard. The filter was designed mainly to reduce the two harmonics on either side of the $403 \mathrm{MHz}$ signal. Using (3) with a Q-Factor of 20, the chosen circuit parameters were $R=$ $30 \Omega, C=0.6 p F$ and $L=180 n H$. After having passed through the bandpass filter, a $300 \mathrm{kHz}$ OOK signal was also passed through the transmitter. The spectrum of the signal received at the external data receiver is presented in Fig. 9, with its time domain in Fig. 10. The signal at $134 \mathrm{MHz}$ 


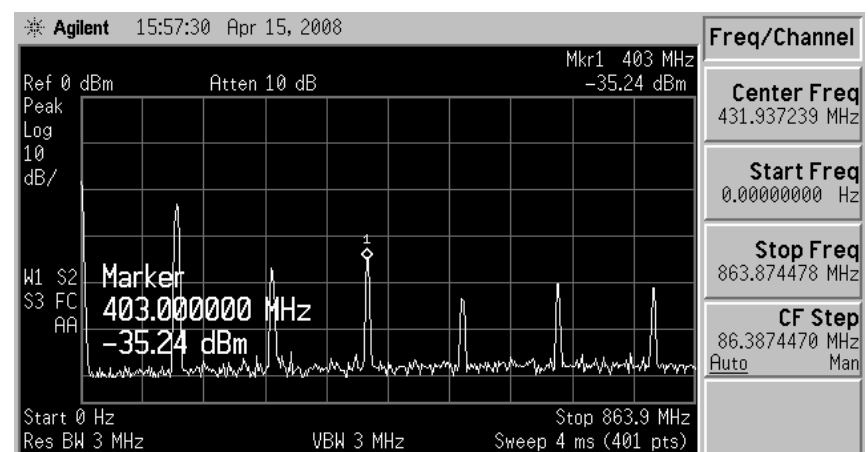

Fig. 8. Spectrum Plot of the Inverter Output

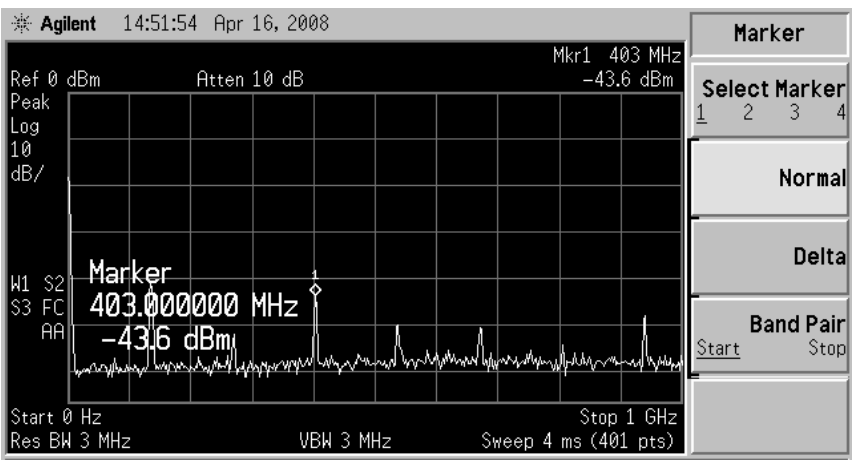

Fig. 9. Signal Spectrum of the Received Data Signal

is in fact interference from the power signal transmission, and it can be easily eliminated at the external data receiver using a simple or complex filter or with a differential method suggested in [9].

\section{E. Inductive Coils and Power Consumption}

Each of the inductive coils were made according to (4), where $r$ is the coil radius, $l$ is the inductor length, $\mu_{0}$ is the permeability of free space, $N$ is the number of coil turns, $\varepsilon_{0}$ is the permittivity of free space and $a$ is the radius of the wire [10]. Where possible, the coils were optimized to self resonate at their desired transmission frequency using their internal capacitance.

The $134 \mathrm{MHz}$ power coils were $10 \mathrm{~mm}$ in diameter with 3

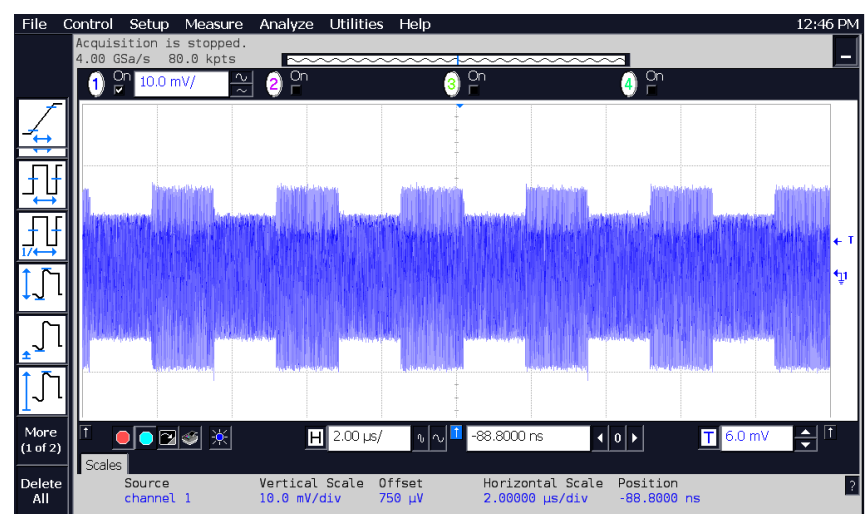

Fig. 10. Received $403 \mathrm{MHz}$ signal with OOK data modulation turns of $0.5 \mathrm{~mm}$ diameter copper wire. They were positioned $1 \mathrm{~cm}$ apart. The $403 \mathrm{MHz}$ data coils were $6 \mathrm{~mm}$ in diameter with 5 turns of $1 \mathrm{~mm}$ diameter wire. They were positioned $3 \mathrm{~cm}$ apart. The power coils were positioned perpendicular to the data coils, and it was noted that filtering would be necessary to avoid interference, especially at the data receiver.

$$
L=\frac{\pi r^{2} \mu_{0} N^{2}}{l}, C=\frac{\varepsilon_{0} .2 \pi r N .2 a}{l / N}
$$

The external power transmitter emitted $10.50 \mathrm{dBm}$ $(11.25 \mathrm{~mW})$, which was enough to entirely power the implantable device including the path loss between the coils. The power level of the $403 \mathrm{MHz}$ signal at the inverter output was $-35.24 \mathrm{dBm}(0.33 \mu \mathrm{W})$ prior to entering the Band Pass Filter Transmitter. At the $403 \mathrm{MHz}$ receiver, the OOK modulated power was $-43.65 \mathrm{dBm}(43.65 \mathrm{nW})$. The techniques used here are based on using passive elements, and it results in an extremely low power system.

\section{CONCLUSION}

This paper proposed a new harmonics-based system that aimed to replace power-hungry frequency blocks with an elegant system which utilized the oscillatory nature of power signals. It showed how a device's power consumption and space can be decreased while still possessing the ability to transmit information with a high frequency.

\section{REFERENCES}

[1] MICS Band Plan, Table of Frequency Regulations, Part 95 ed., FCC Rules and Regulations, 2003.

[2] P. Troyk and M. Schwan, "Closed-loop class e transcutaneous power and data link for microimplants," Biomedical Engineering, IEEE Transactions on, vol. 39, no. 6, pp. 589-599, Jun 1992.

[3] H. Lim, Y. Yoon, C. Lee, I. Park, B. Song, and J. Cho, "Implementation of a transcutaneous charger for fully implantable middle ear hearing device," in IEEE-EMBS 2005. 27th Annual International Conference of the. Engineering in Medicine and Biology Society, 2005, pp. 6813-6816.

[4] P. Li and R. Bashirullah, "A wireless power interface for rechargeable battery operated medical implants," Circuits and Systems II: Express Briefs, IEEE Transactions on [see also Circuits and Systems II: Analog and Digital Signal Processing, IEEE Transactions on], vol. 54, no. 10, pp. 912-916, Oct. 2007.

[5] G. Wang, W. Liu, M. Sivaprakasam, M. Zhou, J. D. Weiland, and M. S. Humayun, "A dual band wireless power and data telemetry for retinal prosthesis," in Engineering in Medicine and Biology Society, 2006. EMBS '06. 28th Annual International Conference of the IEEE, 2006, pp. 4392-4395.

[6] M. D. Zimmerman, N. Chaimanonart, and D. J. Young, "In vivo rf powering for advanced biological research," in 28th Annual International Conference of the IEEE, E. in Medicine and B. Society, Eds., Aug 2006, pp. 2506-2509.

[7] M. Yuce, W. Liu, M. Chae, and J. Kim, "A wideband telemetry unit for multi-channel neural recording systems," in IEEE International Conference on Ultra-Wideband, 2007.

[8] N. Sokal, "Class-e switching-mode high-efficiency tuned rf/microwave power amplifier: improved design equations," Microwave Symposium Digest., 2000 IEEE MTT-S International, vol. 2, pp. 779-782 vol.2, 2000.

[9] M. Zhou, G. Wang, M. Sivaprakasam, M. Yuce, J. Weiland, and M. Humayun, "A transcutaneous data telemetry system tolerant to power telemetry interference," Engineering in Medicine and Biology Society, 2006. EMBS '06. 28th Annual International Conference of the IEEE, pp. 5884-5887, 2006.

[10] R. Ludwig and P. Bratchko, RF circuit design: theory and applications. Prentice Hall, 2000. 